\title{
Iodoform: A boon in disguise
}

\author{
Vibha Singh", Somdipto Das, Nand Kishore Sharma \\ Department of Oral and Maxillofacial Surgery, King George’s Medical University, Lucknow, India \\ Email: "vibhasinghraghuvanshi@gmail.com, "drvibha66@yahoo.com
}

Received 5 September 2012; revised 8 October 2012; accepted 15 October 2012

\begin{abstract}
Iodoform is also known as triiodomethane a yellow crystalline solid belongs to the family of organic halogen compounds used as an antiseptic component of certain medications. It was first prepared in 1822 by electrolysis of aqueous solutions of acetone, inorganic iodides and sodium carbonates. It $s$ antiseptic action discovered in 1880, which made it an important medicinal. It has no irritant action; it has antiseptic, disinfectant properties. In this article we are sharing our experiences of iodoform dressing in different conditions like for the cyst healing and management of dry socket, followed by a review of its various applications in literature. The open packing has been used successfully as a secondary healing method to help bone tissue healing, results were similar to secondary healing of alveolar socket after tooth extraction. Bony healing was confirmed radio graphically. The release of elemental iodine when applied to the tissue which has a mild disinfectant action with the organic tissue, iodine is slight irritant to the tissue which may promote granulation tissue formation.
\end{abstract}

Keywords: Keratocyst; Alveolar Osteitis; Bone Healing

\section{INTRODUCTION}

Iodoform is an organo-iodine compound with chemical formula of $\mathrm{CHI}_{3}$. Iodoform, also known as triiodomethane, is a yellow crystalline solid which belongs to the family of organic halogen compounds used as an antiseptic component for certain medications. It was first prepared in 1822 by electrolysis of aqueous solutions of acetone, inorganic iodides and sodium carbonates. Its antiseptic action was discovered in 1880, which made it an important medicinal compound. It has no irritant action; but with an antiseptic, disinfectant properties. Its beneficial effects on the tissue of the wounds are due to its protecting power both mechanically and maintaining asepsis thus promoting faster healing. It destroys the toxic substances formed by the microorganism. For the ulcerated

\footnotetext{
${ }^{*}$ Corresponding author.
}

wounds it can be mixed with glycerine followed by its application in the form of ribbon gauze. Iodoform is commonly used in dental practice as an antiseptic either alone, or in combination with eucalyptus oil or glycerine for the treatment of dry socket, as a dressing for the large cystic defects and for the soft tissue dressing in the cases of dehiscence of wounds or to promote healing by secondary intention.

George Serrulas and John Thomas Cooper, working independently, both had been accredited with the discovery and preparing iodoform as early as in 1822 [1].

Amongst the various combinations in which it's dispensed for application, some of them are specified viz;

1) Iodoform impregnated in ribbon gauze (Figure 1).

2) Iodoform in combination with benzoin, prepared storax, balsam of tolu as "whitehead's varnish".

3) Iodoform as a component of Bismuth Iodine Paraffin Paste (BIPP).

\section{MATERIALS AND METHODS}

In this study we are going to present our experiences of using iodoform dressing in different. Clinical situations and it was observed that it plays a wonderful role in healing of the cyst and alveolar osteitis. Iodoform dressing is commonly used in our clinical practice at our Institute so there is no need of taking ethical approval.

In our study, we treated 24 patients (Male:Female: 17:7) ranging from 15 - 70 years, diagnosed of odonto-

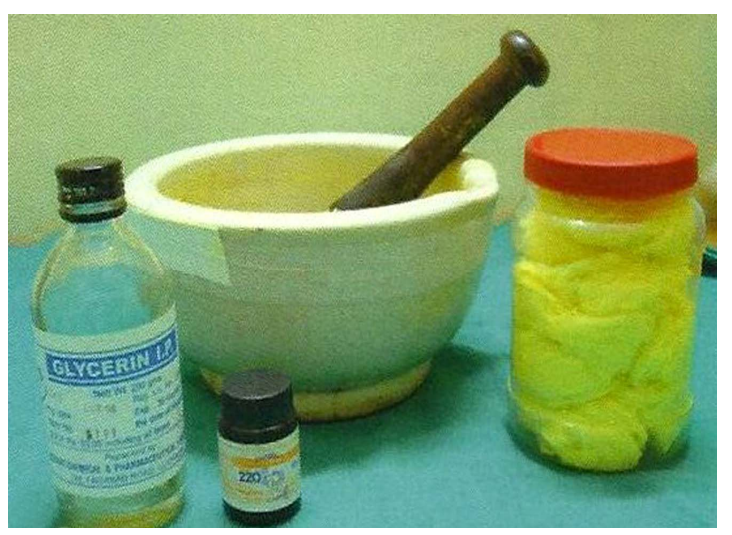

Figure 1. Iodoform dressing. 
genic keratocyst primarily on the basis of clinical, radiographic and histological reports. Patients with history of recurrence of lesion were excluded from the study. All patients were informed about their disease and written consent was taken from them. Patients were treated under local anesthesia (lignocaine hydrochloride.) on outpatient basis. A buccal vestibular incision was given which was deep enough to remove off the superficial lining of cyst wall and overlying mucosa which was later sent for histopathological examination for reconfirmation. Rotary instrument was used for removing irregular bony surface to prevent recurrences due to satellite cysts. The overlying soft tissues above the bony window is excised as Stoelinga (1971, 2001, 2003), Stoelinga and Peters (1973) [2] in a number of publications demonstrated convincingly that $\mathrm{OKC}$ can arise from basal cells of oral mucosa in the region of third molar and ascending ramus. Suturing was done all around the soft tissue window involving the mucosa and cystic lining. Iodoform gauze was placed into the cavity and changed twice a week. Follow up was done between 30 - 36 months. Bone healing was confirmed periodically with the help of radiograph (Figures 2 and 3) (Table 1).

In this study we included 50 patients (Male:Female: $1: 2$ ) between the age group of 15 - 60 years were treated in the outpatient department in our institute as a case of alveolar osteitis; popularly known as “dry socket”. Diagnosis of dry socket was done mainly by clinical presentation of patients symptoms like history of excruciating pain which arose second or third day following the extraction and referral of pain over the ear and temporal region. Clinically all patients showed presence of greyish material within socket which dislodged on irrigation with warm normal saline. The sockets were denuded of blood clot with pain on probing the exposed bone. All patients also had a history of taking non steroidal analgesics but with no relief which also confirmed our diagnosis. The patients were clinically evaluated and were informed of the condition. Written consent was taken from them of the procedure. The sockets were irrigated with normal saline and curettage was avoided. Iodoform ribbon gauze was applied over the socket avoiding tight packing. All patients were given supplemental analgesics and recalled everyday to change the pack as this foreign substance can entrap food substances and could be a source of superimposed infection. Follow up was done up to 14 days with all patients relieved of pain within 3 - 5 days after application of iodoform gauze (Table 2).

\section{DISCUSSION}

Bony defects in the jaws are frequently observed because odontogenic cysts and tumours are common entity. Patients do not seek treatment either because of their small size which gets frequently gets revealed only after routine radiographs or because of not eliciting pain. The success of the bone healing is directly related to the size of bony defects, anatomical location, age of the patient, vascularity of the surrounding bone and infection among other parameters [3]. There have been various treatment

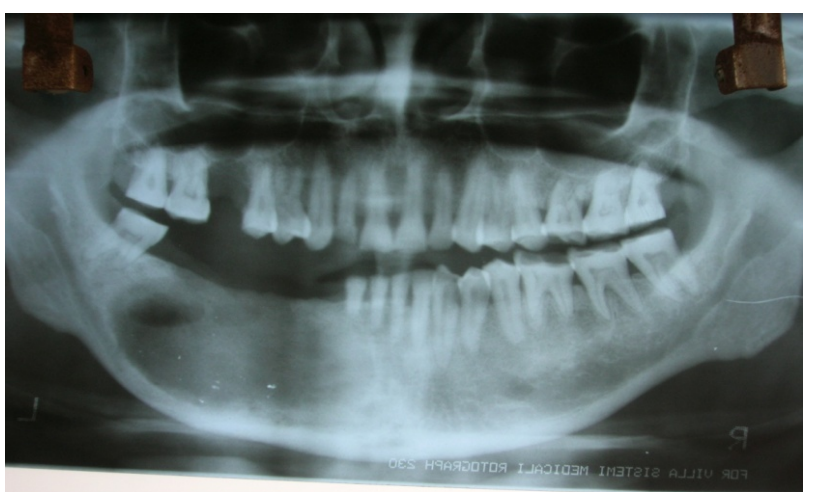

Figure 2. Post operative Radiogrph showing complete bone formation in a case of odontogenic keratocyst.

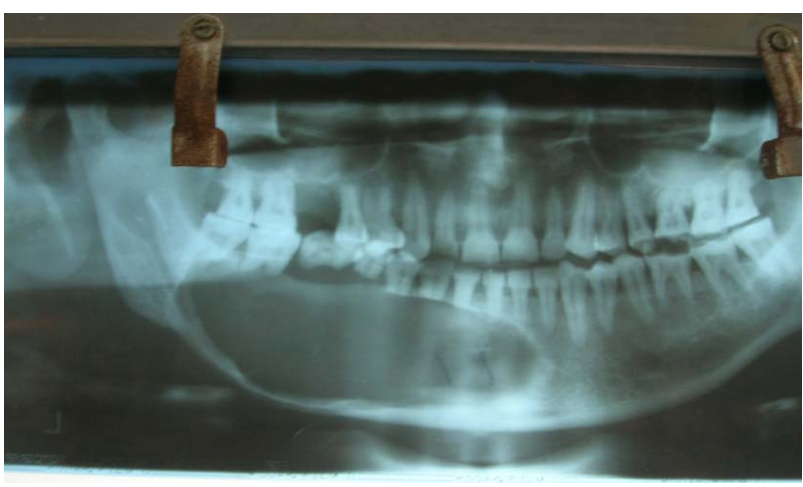

Figure 3. Pre-operative radiograph showing radiolucent lesion.

Table 1. Table showing treatment modalities and recurrence in cases of Odontogenic keratocyst.

\begin{tabular}{ccccc}
\hline Age group (years) & Number of patients & Treatment given & Recurrence \\
\hline $0-20$ & 11 & Curettage followed by iodoform dressing & 1 & Resection and reconstruction with iliac crest graft. \\
$20-30$ & 6 & Curettage followed by iodoform dressing & 0 \\
$30-50$ & 6 & Curettage followed by iodoform dressing & 0 \\
$50-70$ & 1 & Curettage followed by iodoform dressing & 0 \\
$70-$ above & 0 & & \\
\hline
\end{tabular}


Table 2. Showing patients with dry socket.

Days Number of patients with necrotic slough Patients with trismus Dressing given Iodoform dressing Irrigation solution Povidon iodine solution

\begin{tabular}{lllll}
\hline 1 & 50 & 12 & - & - \\
2 & 50 & 12 & - & - \\
3 & 43 & 12 & - & - \\
4 & 7 & - & - & - \\
5 & 35 & 5 & - & - \\
6 & 33 & 1 & - & - \\
7 & 0 & - & - \\
8 & 30 & - & - & - \\
9 & 21 & - & - & - \\
10 & 1 & - & - & - \\
11 & - & - & - & - \\
13 & - & - & - & -
\end{tabular}

There was no trismus after 6 days in any of the patients. There was no necrotic slough present after 9 days.

modalities which are reported in the literature for the management of OKC. The treatment modalities range from conservative management which includes marsupialisation, nucleation and curettage whereas radical spectrum of surgical treatment includes resection with or without continuity defect and disarticulation [4], Because OKC occur over a wide age range, cases as early as first decade been reported in literature [2] several studies demonstrated bimodal age distribution with first peak in second and third decade and second in fifth decade or later [5]. OKC is always described in literature as most aggressive lesions which have a strong tendency to recur [6]. The first to report the aggressive behaviour were Pindborg and Hansen (1963) where they found recurrence rate of $62 \%$ in their study of 16 cases. But recently Stoelinga (2001) did a study on 49 cases where marsupialisation was done followed by excision of overlying mucosa and application of Carnoy's solution found recurrence rate of $6 \%[7,8]$.

Similar studies was done also by Giuliani(2006) where he reported of large OKC over mandible treated by marsupialisation and iodoform dressing followed by enucleation with no recurrence of the lesion.

Zhou (2012) studied [9] patients with OKC's where enucleation was done followed by iodoform dressing with reported case of 1 recurrence over a follow up of 6 years.

In our institute, we followed all the patients over duration of 30 - 36 months without any recurrence and complete uneventful healing except in one patient.

Alveolar osteitis on other hand refers to a very unpleasant local complication following surgical removal of the tooth. It is synonymously known by various terms like alveolologia [10,11], dolor post extraction [12], leeren alveoli [13], necrotic alveolar socket, alveolar sicca dolorosa, epithelialised socket and painful socket [14]. It is also termed in terms of localised osteomyelitis as an acute alveolar osteomyelitis of the jaw [15]. Pain is one of the chief complaints of the patient which makes the patient seek treatment. Besides pain, inflammation around the marginal gingiva surrounding the unhealed socket, halitosis and complain of a bad taste in mouth are other complains [5].

Joseph Schroff (1929) used iodoform dressing for packing the socket and found immediate reduction of pain overnight. There has been enumerable ways used for its management as reported in the literature. It includes zinc oxide eugenol paste, iodoform gauze, and antibiotics [17]. Iodine is a known antiseptic and germicide which has numerous clinical applications mainly for infected wounds. Its clinical spectrum includes vegetative pathogens including anaerobic bacteria within the oral cavity, viruses, fungi, and protozoan. Iodide is also found effective against vegetative spores. Since anaerobic bacteria of the oral cavity includes streptococcus facultative, porphyromonas, prevotella, peptostreptococci are causative of dry socket [16]. Iodide released from iodoform is a suitable agent applied after irrigating the socket with warm saline.

Iodide in general follows the criteria as laid down by Mitchell [17] (1986) as an ideal dressing material for dry socket: which promotes fast and effective relief of pain, non irritating, easily absorbed, antiseptic, stable to oral fluids and blood makes it a perfect dressing material. But it's also not without any complications. Hypersensitivity to iodine can be a complication which can be avoided by proper history of the patient and by skin patch test. Similarly neurotoxicity due to absorption of bismuth 
from BIPP pack is rare but a preventable and reversible complication but likely to be fatal if unrecognised. Blood bismuth concentration may increase correlating well with clinical state [18]. Similarly dermatitis herpetiformis which is an autoimmune blistering disease was flared following the exposure of triiodomethane which was used as a packing strip for alveolar osteitis [15].

\section{CONCLUSION}

Iodine and iodoform mainly acts as an antiseptic which promotes healing by asepsis. Hence from our study, we conclude that iodoform dressing is an excellent tool for the management of odontogenic keratocyst and dry socket.

\section{REFERENCES}

[1] Iodoform (2012) Encyclopædia Britannica. Encyclopædia Britannica Online. Encyclopædia Britannica Incorporated Webside, 30 March 2012.

[2] Stoelinga, P.J.W. (2001) Long-term follow-up on keratocysts treated according to a defined protocol. International Journal of Oral and Maxillofacial Surgery, 30, 14-25. doi:10.1054/ijom.2000.0027

[3] Maurette, P.E., Jorge, J. and de Moraes, M. (2006) Conservative treatment protocol of odontogenic keratocyst: A preliminary study. Journal of Oral and Maxillofacial Surgery, 64, 379-383.

[4] Enislidis, G., Fock, N., Sulzbacher, I. and Ewers, R. (2004) Conservative treatment of large cystic lesions of the mandible: A prospective study of the effect of Decompression, British Journal of Oral and Maxillofacial Surgery, 42, 546-550.

[5] Jones, A.V., Craig, G.T. and Franklin, C.D. (2006) Range and demographics of odontogenic cysts diagnosed in a UK population over a 30-year period. Journal of Oral Pathology and Medicine, 35, 500-507. doi:10.1111/j.1600-0714.2006.00455.X

[6] Nakamura, N., Mitsuyasu, T., Mitsuyasu, Y., Taketomi, T., Higuchi, Y. and Ohishi, M. (2002) Marsupialization for odontogenic keratocysts: Long-termfollow-up analysis of the effects and changes in growth characteristics. Oral Surgery, Oral Medicine, Oral Pathology, Oral Ra- diology and Endodontology, 94, 543-553. doi:10.1067/moe.2002.128022

[7] Ihan Hren, N. and Miljavec, M. (2008) Miljavec Spontaneous bone healing of the large bone defects in the mandible. International Journal of Oral and Maxillofacial Surgery, 37, 1111-1116. doi:10.1016/j.ijom.2008.07.008

[8] Stoelinga, P.J.W. (2001) Stoelinga Long-term follow-up onkeratocysts treated accordingto a defined protocolInt. International Journal of Oral and Maxillofacial Surgery, 30, 14-25. doi:10.1054/ijom.2000.0027

[9] Zhou, H., Hou, R., Ma, Q., Wu, K., Ding, Y., Qin, R. and $\mathrm{Hu}, \mathrm{K}$. (2012) Secondary healing after removal of large keratocystic odontogenic tumor inthe mandible: Enucleation followed byopen packing of iodoform gauze. Journal of Oral and Maxillofacial Surgery, 70, 1523-1530.

[10] Crawford, J.Y. (1896) Dry socket. Dental Cosmos, 38, 929-931.

[11] Bjercke, B. (1960) Dry socket. En oversikt. Nor. Tannlaegeforen. Tid., 70, 153-163.

[12] Partsch, C. (1892) Uber Kiefercysten. Dtsch Mschr Zahnheilkd, 10, 271.

[13] Partsch, C. (1910) Zur Behandlung der Kieferzysten. Dtsch Mschr Zahnheilkd, 28, 252.

[14] Mitchell, D.A. (1988) Nitroimidazole for alveolar osteitis. Journal of Oral and Maxillofacial Surgery, 46, 720. doi:10.1016/0278-2391(88)90176-0

[15] Cardoso, C.L., Rodrigues, M.T.V., Júnior, O.F., Garlet, G.P. and de Carvalho, P.S.P. (2010) Clinical Concepts of Dry Socket. Journal of Oral and Maxillofacial Surgery, 68, 1922-1932. doi:10.1016/j.joms.2009.09.085

[16] Katz, K.A., Roseman, J.E., Roseman, R.L. and Katz, S.I. (2009) Dermatitis herpetiformis flare associated with use of triidomethane (Iodoform) packing strips for alveolar osteitis. Journal of the American Academy of Dermatology, 60, 352-353.

[17] Mitchell, D.A. (1988) Nitroimidazole for alveolar osteitis. Journal of Oral and Maxillofacial Surgery, 46,720. doi:10.1016/0278-2391(88)90176-0

[18] Sharma, R.R., Cast, I.P., Redfern, R.M. and O’Brien, C. (1994) Extradural application of bismuth iodoform paraffin paste causing relapsing bismuth encephalopathy: A case report with CT and MRIstudies. Journal of Neurology, Neurosurgery, and Psychiatry, 57, 990-993. 